\author{
Ewa GAJDA-ZaGÓRSKA \\ MACIEJ PASZYŃSKI \\ ROBERT SCHAEFER \\ DAVID PARDO
}

\title{
HP-HGS STRATEGY FOR INVERSE AC/DC RESISTIVITY LOGGING MEASUREMENT SIMULATIONS
}

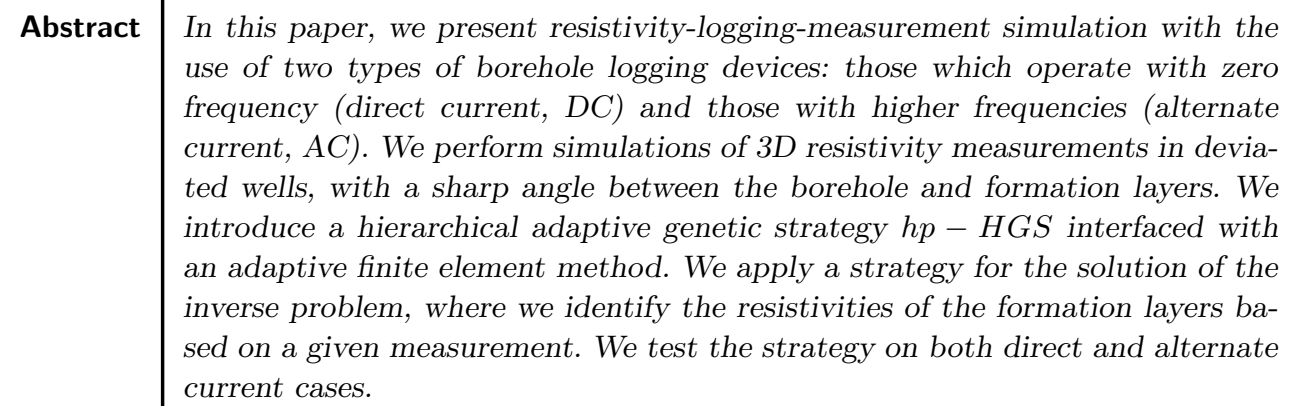




\section{Introduction}

Simulations of resistivity measurements are widely used in geophysics where a quantity of interest, voltage, is measured at a receiver electrode located in the logging instrument. In 3D-resistivity-logging measurements, logging instruments have several transmitter and receiver electrodes. Instruments move along the borehole axis and measure voltage induced at receiver electrodes at different positions. Voltage measured at receivers is proportional to electrical conductivity. Thus, logging instruments estimate properties (electrical conductivity) of the sub-surface material. The ultimate goal is to describe hydrocarbon-bearing formations (with both oil and gas).

In this paper, we focus on two types of borehole logging devices: those which operate with very low frequencies (close to 0 ), which are numerically modeled as zerofrequency (direct current, DC), and those with higher frequencies (alternate current, $\mathrm{AC})$. We perform simulations of $3 \mathrm{D}$ resistivity measurements in deviated wells, with an angle between the borehole and formation layers $<90^{\circ}$ (see Fig. 1, 2).

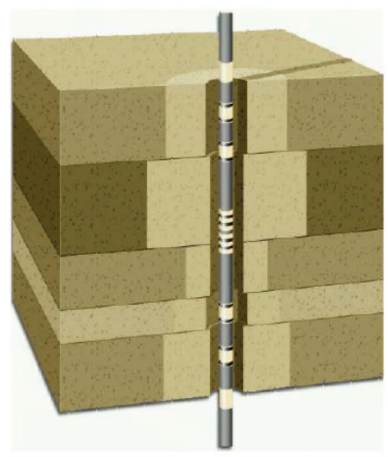

(a) Straight well.

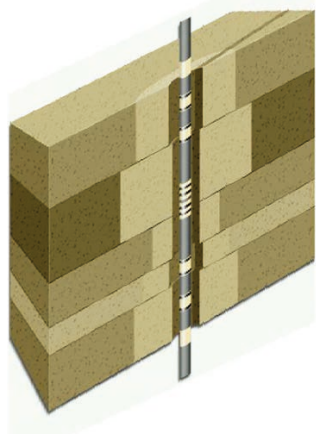

(b) Deviated well.

Figure 1. Boreholes.

Exemplary process of resistivity measurements (with the usage of a logging tool) is presented in Figure 1. The left panel illustrates formation layers that are perpendicular to the borehole. The right panel illustrates a deviated well with formation layers located under an angle with respect to the borehole. The logging tool used during resistivity logging measurements is presented in Figure 2. The logging tool is shifted along the borehole. The transmitter electrode generates electromagnetic waves which are reflected by formation layers. The reflected waves are recorded at the receiver electrodes. For each position of the logging tool, we record two values at two receiver electrodes. We compute the approximation to the first derivative of the scalar potential using a finite difference scheme, and the two values recorded at receiver electrodes. The result is an approximated value of the voltage. The value of the voltage is recorded for each position of the logging tool. In the DC case, this data has real values; in 


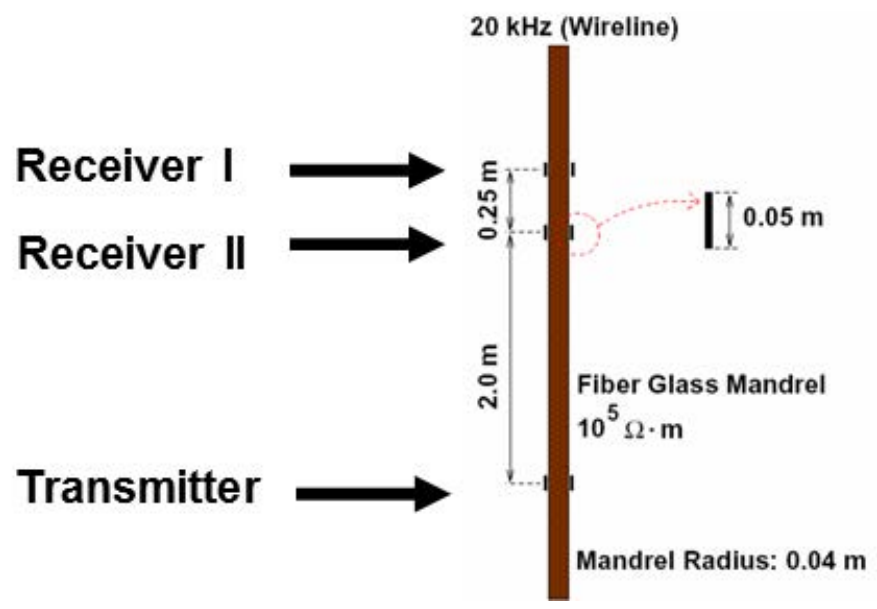

Figure 2. Tool used for 3D AC resistivity logging measurements.

the AC case, this data has complex values. The recorded voltage monotonically depends on the electrical conductivity of the sub-surface material. Thus, the formation layers are represented by the electrical conductivities. The exemplary distribution of conductivities in several formation layers is presented in the right panel in Figure 3. The resulting sequence of values recorded for the $\mathrm{AC}$ case is presented in the middle and right panels in Figure 3. There are 3 logging curves which correspond to the three assumed situations, where formation layers are perpendicular to the borehole (0 degrees case), and the deviated well case (either 30 or 60 degrees).

There are several numerical simulation methods developed to improve the interpretation of results obtained with resistivity measurements, and thus, to better quantify and determine existing subsurface materials and increase hydrocarbon recovery (i.e., $[19,25,8,12,5,23,24,3])$. A second group of methods can be utilized to invert well-log measurements $[1,2,11]$.

A combination of a numerical method based on a Fourier series expansion in a non-orthogonal system of coordinates with a $2 \mathrm{D}$ self-adaptive $h p$ goal-oriented Finite Element (FE) method [14, 16, 17, 15] was described in [13, 18]. This FourierFinite-Element method was formulated and applied to direct and alternating current resistivity logging problems, and it enabled fast and accurate simulations of previously-unsolved EM simulation problems in deviated wells.

In this paper, we consider two types of problems. First, we focus on the forward problem, which consists of finding the voltage for a sequence of transmitter positions and receiver electrodes given the known conductivities of formation layers. By solving the forward problem for consecutive positions of the logging tool, we generate a sequence of points (either real- or complex-valued, for DC or AC formulations respectively), ultimately forming a logging curve. Second, we focus on the inverse 

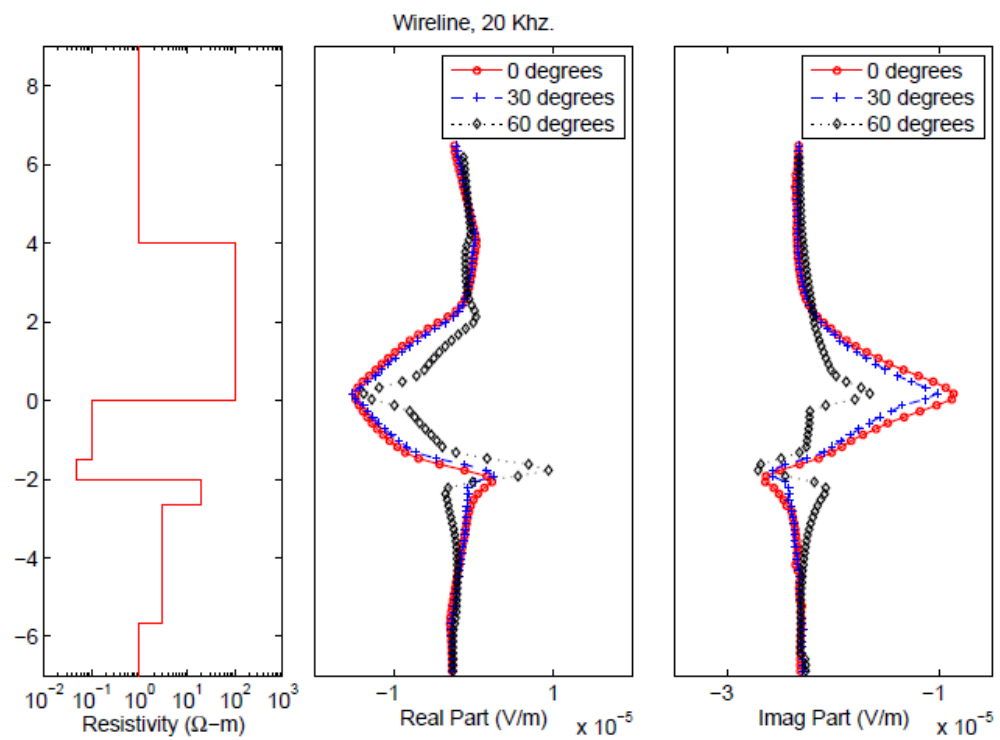

Figure 3. Resistivities of formation layers generating the complex logging curve.

problem of global optimization where, for a given reference logging curve, we search for conductivities of the formation layers that will result in a similar logging curve.

\section{Forward problem}

Input for the forward problem are the locations of the formation layers, the geometry of the logging tool, the conductivities of formation layers, and the angle between the borehole and the formation layers. Input from the forward problem is a sequence of points generated by using computer simulations. Actually, in the forward problem, we solve a sequence of finite element method problems which differ by location of the transmitter and receiver electrodes (compare Figure 5).

\subsection{Formulation of $3 D D C$ the problem}

At direct current (DC, $f=0$ ), the electromagnetic phenomena governed by Maxwell's equations reduces to the so-called conductive media equation

$$
\nabla \cdot(\sigma \nabla u)=-\nabla \cdot \mathbf{J}^{i m p}
$$

where $\boldsymbol{\sigma}>0$ is the conductivity tensor, $\mathbf{J}^{i m p}$ represents the prescribed, impressed electric current source, and $u$ is the scalar electric potential such that $\mathbf{E}=-\nabla u$. The $J^{i m p}$ is non-zero at transmitter electrodes, and its position changes for each location of the transmitter electrodes. 
By multiplying equation (1) by test function $v \in H_{0}^{1}(\Omega)=\left\{u \in H^{1}(\Omega):\left.u\right|_{\Gamma_{D}}=\right.$ $0\}$, and by integrating by parts over the domain $\Omega$, which is a skewed cylinder with 0 Dirichlet boundary condition (BC), we obtain the following variational formulation:

$$
\left\{\begin{array}{l}
\text { Find } u \in H_{0}^{1}(\Omega) \text { such that: } \\
\langle\nabla v, \boldsymbol{\sigma} \nabla u\rangle_{L^{2}(\Omega)}=\left\langle v, \nabla \cdot \mathbf{J}^{i m p}\right\rangle_{L^{2}(\Omega)} \quad \forall v \in H_{0}^{1}(\Omega),
\end{array}\right.
$$

$\Omega \subset R^{3}$ is assumed to be a simple connected, bounded domain with the Lipshitz boundary (see $[7,6,9]$ for detailed mathematic description). In our case $\Omega$ is a $3 \mathrm{D}$ skewed cylinder surrounding the borehole (see Figures 4, 5).

Thus, the input to our DC forward problem is the geometry of the domain, the angle between formation layers and the borehole, the location of formation layers forming the cylinder $\Omega$, skewed for the case of deviated well, the geometry of the logging tool, the locations of the transmitter and receiver electrodes, as well as the conductivities of formation layers. The $\mathbf{J}^{i m p}$ represents the transmitter electrodes (it is non-zero over a small part of the domain representing the transmitter electrodes).

We solve a sequence of fully 3D problems, which differ by the location of the transmitter and receiver electrodes. From the solution of each problem, we extract the values of the scalar potential at two receiver electrodes and compute the approximation to the voltage by taking the finite difference between the two values. The solution to the forward problem is the logging curve obtained by solving the forward problem (2) for consecutive positions of the logging tool. Each point from the logging corresponds to a single position of the receiver electrodes.
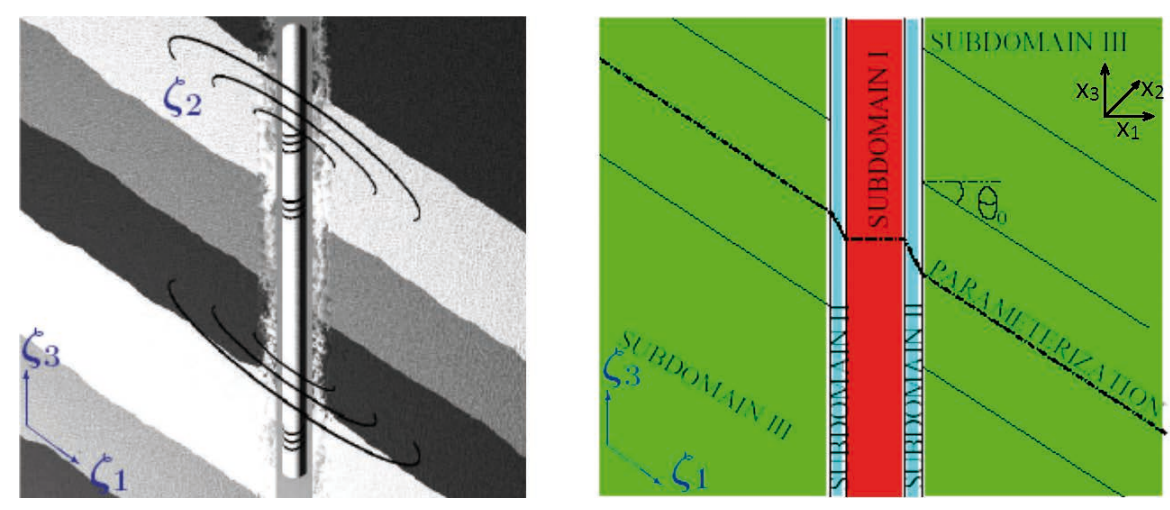

Figure 4. A cross section showing the $3 \mathrm{D}$ geometry of a logging instrument in a vertical well penetrating dipping layers. $\mathbf{x}=\left(x_{1}, x_{2}, x_{3}\right)$ represents the Cartesian system of coordinates, and $\boldsymbol{\zeta}=\left(\zeta_{1}, \zeta_{2}, \zeta_{3}\right)$ represents the new non-orthogonal system of coordinates. The new system of coordinates is different in each of the three sub-domains. Sub-domain I corresponds to the logging instrument, sub-domain II to the borehole, and sub-domain III to the formation. The new system of coordinates is globally continuous, as indicated by the parameterization. 

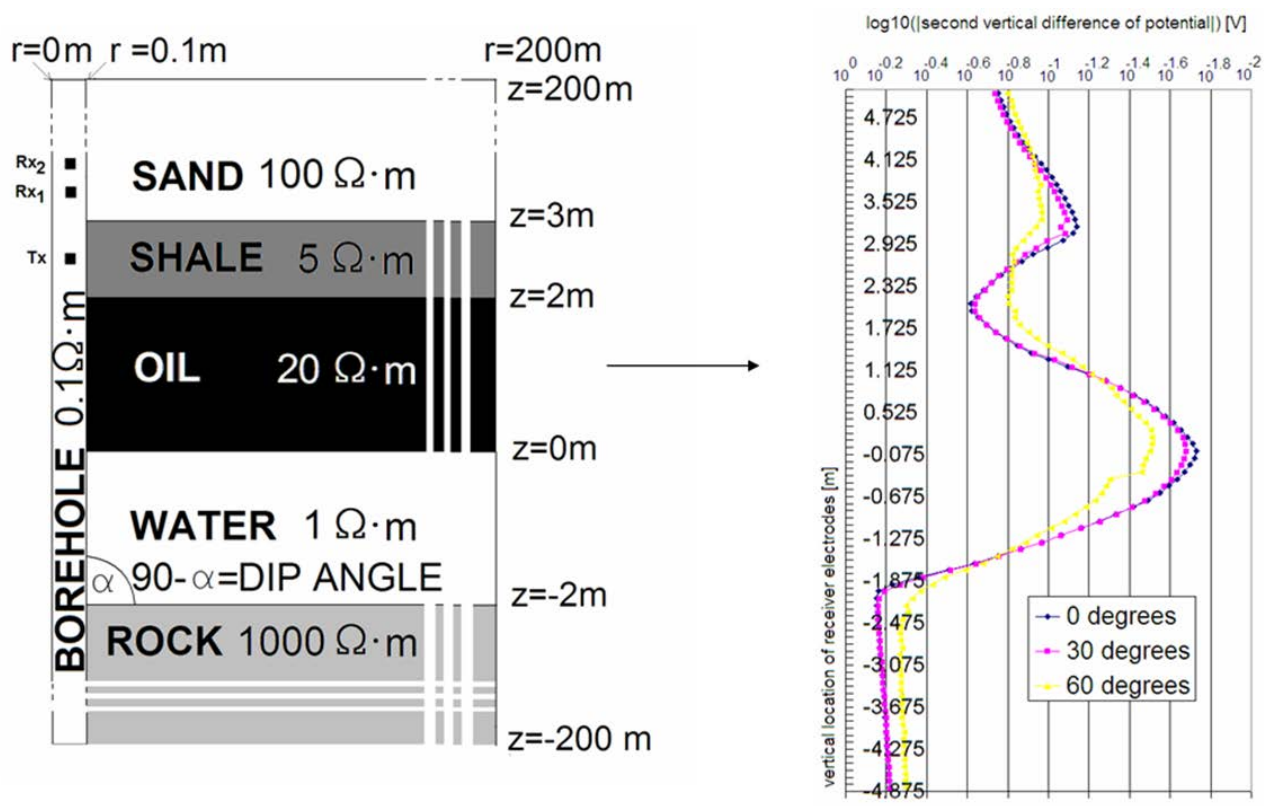

Figure 5. The forward problem consists in computations of the logging curves with assumed resistivities of the borehole and five formation layers.

\subsection{Formulation of $3 D A C$ problem}

We start from the time-harmonic Maxwell's equations with the angular frequency $f \neq 0$

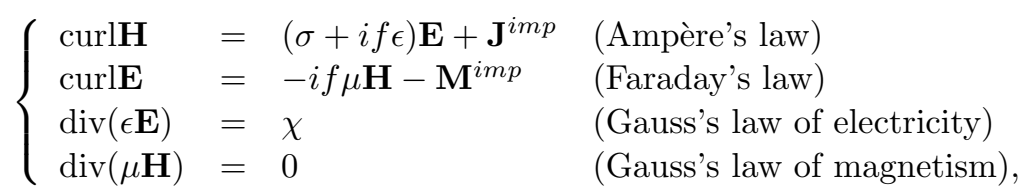

here $\mathbf{H}$ is the magnetic field intensity, $\mathbf{E}$ is the electric field, $\mathbf{J}^{i m p}$ is the density of electic current impressed on transmitter antennas, $\mathbf{M}^{i m p}$ is a density of an impressed magnetic current, $\chi$ is the electric charge distribution and $\epsilon, \mu, \sigma$ stand for the permittivity, permeability, and electrical conductivity of the borehole and formation layers. The geometry of the borehole, the logging tool, and the formation layers for the AC formulation are summarized in Figure 6.

We are looking for solutions to (3) in the 3D skewed cylinder $\Omega \subset \mathbf{R}^{3}$. We assume zero Dirichlet boundary condition on the entire $\Gamma=\partial \Omega$.

The weak variational formulation is considered in

$$
H(\operatorname{curl} ; \Omega)=\left\{\mathbf{F} \in L^{2}\left(\Omega ; C^{3}\right): \operatorname{curl} \mathbf{F} \in L^{2}\left(\Omega ; C^{3}\right)\right\}
$$


endowed with an inner product

$$
(\mathbf{E}, \mathbf{F})_{H(\operatorname{curl} ; \Omega)}=\int_{\Omega} \operatorname{curl} \mathbf{E} \cdot \operatorname{curl} \overline{\mathbf{F}} d x+\int_{\Omega} \mathbf{E} \cdot \overline{\mathbf{F}} d x
$$

where $\mathbf{u} \cdot \mathbf{v}=u_{1} v_{1}+u_{2} v_{2}+u_{3} v_{3}$.

The weak form (the so called $\mathbf{E}$-formulation) is obtained by dividing both sides of Faraday's law by $\frac{1}{\mu}$, multiplying them by curl $\overline{\mathbf{F}}$, integrating by parts over the domain $\Omega$ and finally applying Ampére's law: find $\mathbf{E} \in V$ such that

$$
\int_{\Omega} \frac{1}{\mu} \operatorname{curl} \mathbf{E} \cdot \operatorname{curl} \overline{\mathbf{F}} d x-\int_{\Omega}\left(f^{2} \epsilon-i f \sigma\right) \mathbf{E} \cdot \overline{\mathbf{F}}=-i f \int_{\Omega} \mathbf{J}^{i m p} \cdot \overline{\mathbf{F}} d x
$$

for every test field $\mathbf{F} \in V$. For more details on the problem derivation, we refer to $[18]$.

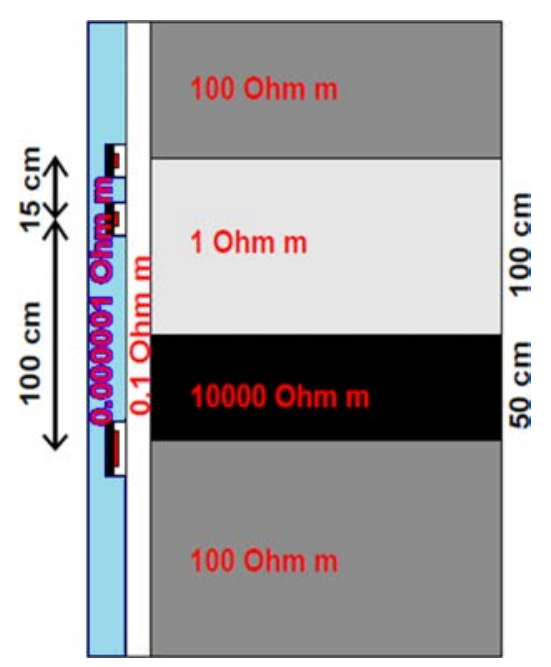

Figure 6. The geometry of the borehole, logging tool, and formation layers for the AC problem.

Thus, the input to our AC forward problem is the geometry of the domain, the angle between formation layers and the borehole, the location of formation layers forming the cylinder $\Omega$, skewed for the case of a deviated well, the geometry of the logging tool, the locations of the transmitter and receiver antennas, the frequency of the tool $f=20 \mathrm{Khz}$, and the conductivities $\sigma$ of the formation layers. The electric current $\mathbf{J}^{i m p}$ is non-zero over the transmitter antennas.

We solve a sequence of fully-3D problems, which differ due to the location of the transmitter and receiver antennas. From the solution of each problem, we extract the complex values of the scalar potential at two receiver electrodes and compute the approximation to the voltage by taking the finite difference between the two 
complex values. The solution to the forward problem is the logging curve obtained by solving the forward problem (6) for the consecutive positions of the logging tool. Each complex valued point from the logging corresponds to a single position of the receiver electrodes.

\subsection{Non-orthogonal system of coordinates}

The direct problem is formulated for a $3 \mathrm{D}$ skewed cylinder; therefore, we perform a change of variables from Cartesian to non-orthogonal system of coordinates (see Fig. 4). First, we consider the union of three (possibly rotated) cylindrical systems of coordinates defined over sub-domains I, II, and III, respectively, as illustrated in Fig. 4. The change of coordinates $\zeta=\left(\zeta_{1}, \zeta_{2}, \zeta_{3}\right)=\psi(\mathbf{x})$ is globally continuous and with positive Jacobian $J$, therefore suitable for Finite Element computations.

In addition, we observe that $J$, as a function of $\zeta_{2}$, can be represented as a linear combination of functions $1, \sin \zeta_{2}$, and $\cos \zeta_{2}$ because the change of coordinates is composed of rotations of the cylindrical system of coordinates. It is easy to see that the corresponding metric $G=J^{T} J$ (as a function of $\zeta_{2}$ ) can be represented in terms of the following five Fourier basis functions: $1, \cos \zeta_{2}, \sin \zeta_{2}, \cos ^{2} \zeta_{2}$, and $\sin ^{2} \zeta_{2}$. Since material properties for the geometry described in Fig. 4 (deviated wells) are constant with respect to the new quasi-azimuthal direction $\zeta_{2}$, and the metric can be represented exactly with only five Fourier modes, we conclude that, when using a 1D Fourier series expansion in terms of $\zeta_{2}$, the corresponding stiffness matrix becomes penta-diagonal (as opposed to a dense matrix) with respect to $\zeta_{2}$, leading to a dramatic reduction on the computational complexity. For details, see [13].

\subsection{Self-adaptive goal-oriented $h p$ finite element method}

The forward problem has been solved with self-adaptive, goal-oriented $h p$ finiteelement method ( $h p$-FEM). The algorithm starts with an initial mesh, called the coarse mesh and solves the weak problem (either (2) or (6) for DC or AC, respectively). The mesh is then globally $h p$-refined, each element is broken into four elements, and the polynomial order of approximation is increased by one. The resulting mesh is called the fine mesh.

The weak problem is solved again over the fine mesh. The algorithm considers different refinement strategies for each finite element from the coarse mesh. The corresponding local relative error estimations are obtained by projecting the fine mesh solution into the proposed local refinement and comparing the error between the refined and coarse element.

The goal-oriented algorithm incorporated the error estimators aiming to minimize the error at the receiver antenna. For more details on the algorithm, we refer to [15]. The optimal refinements are selected and executed locally for each finite element from the coarse mesh. The resulting optimal mesh becomes the coarse mesh for the next iteration. The iterations are repeated until the required accuracy at the receiver antennas is reached. 


\section{Inverse problem}

For a given reference logging curve, the geometry of formation layers and the resistivity field $\omega=\frac{1}{\sigma}$ of the borehole and top and bottom formations, assuming the constant resistivity in each layer, we search in the DC case for a discrete function $\omega=\left(\omega_{0}, \omega_{1}, \omega_{2}\right)$ (see Figure 7 ). The inverse problem will be formulated as a globaloptimization one. We are looking for each global minimizer $\hat{\omega} \in \mathcal{D} \subset R^{3}$ such that the corresponding computed logging curve is closest to the reference one among all logging curves associated with $\omega \in \mathcal{D}$, where $\mathcal{D}$ stands for the set of admissible resistivities.

The reference logging curve is usually obtained from field measurements. For testing purposes, we compute this curve for the 60 degrees deviated well by using a self-adaptive goal oriented $h p$-FEM algorithm with high accuracy $10^{-5}$. There are: a borehole with resistivity $0.1 \Omega \cdot m$, sand with resistivity $100 \Omega \cdot m$, shale with resistivity $5 \Omega \cdot m$, oil with resistivity $20 \Omega \cdot m$, water with resistivity $1 \Omega \cdot m$ and rock with resistivity $1000 \Omega \cdot m$, which makes five layers as in Figure 7 .

In the case of $\mathrm{AC}$, measurements are much more complex; therefore, the problem was simplified to two parameters: $\omega=\left(\omega_{0}, \rho_{1}\right)$. Global minimizers are of form $\hat{\omega} \in$ $\mathcal{D} \subset R^{2}$. Resistivity of the mandrel equals $10^{-6} \Omega \cdot m$, resistivity of the borehole equals $0.1 \Omega \cdot m$ and there are four formation layers: sand with resistivity $100 \Omega \cdot m$, water with resistivity $1 \Omega \cdot m$, rock with resistivity $10^{4} \Omega \cdot m$ and sand with resistivity $100 \Omega \cdot m$ as in Figure 6.

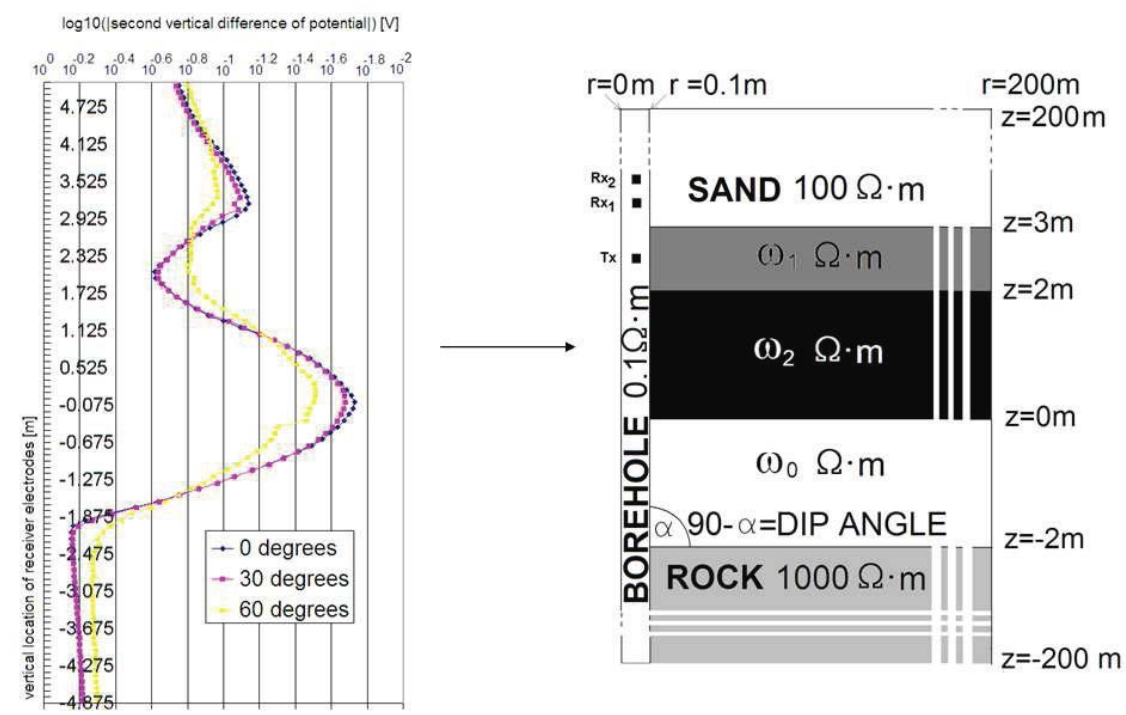

Figure 7. The inverse problem is to find resistivities of formation layers from a given logging curve. Three layers are sought in DC case. 


\section{Strategy}

\subsection{Hierarchic Genetic Strategy}

Hierarchic Genetic Strategy (HGS) produces a tree-structured set of concurrent evolutionary processes (see Figure 8). The strategy was introduced by Kołodziej and Schaefer in [21]. The structure of the tree changes dynamically and its depth is bounded by $m<+\infty$. We will focus on the case in which each evolutionary process is governed by the Simple Genetic Algorithm [22] with proportional selection, one-point crossover and binary mutation with Bernoulli distribution.

$m=3$

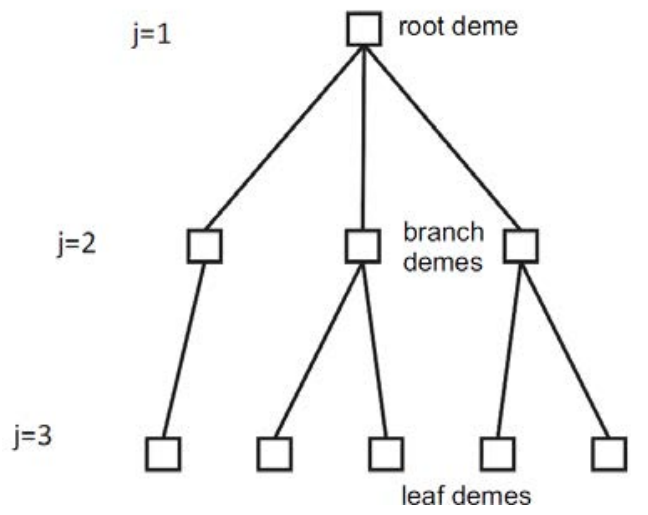

(9)
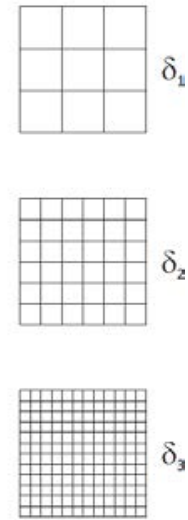

Figure 8. HGS tree and corresponding coding meshes.

HGS starts with a single root deme (population) which is a first order process. Root performs a chaotic search with low accuracy. After a fixed number of genetic epochs $K$ called the metaepoch, the root-deme sprouts a child-deme in the promising region of the evolutionary landscape surrounding the best-fitted individual distinguished from the parental-deme (see left panel in Figure 9). Demes at the second level (called branches) perform more local search with higher accuracy. Third-order demes (leaves) are sprouted by branches similarly as branches are sprouted by root-demes (see middle panel in Figure 9). Leaves perform local and most accurate search (see right panel in Figure 9). In the second metaepoch, evolution is performed in all existing populations and new demes are sprouted. The algorithm continues until the global stop condition is reached.

HGS implements two mechanisms that prevent redundancy in the search: conditional sprouting and branch reduction. The former allows new demes to be sprouted only in regions which are not explored by sibling-demes (demes sprouted by the same parent). The latter reduces (kills) demes of the same order that perform searches in common landscape regions or in those regions already explored. A population is also killed when its average fitness does not change in several consecutive epochs. 

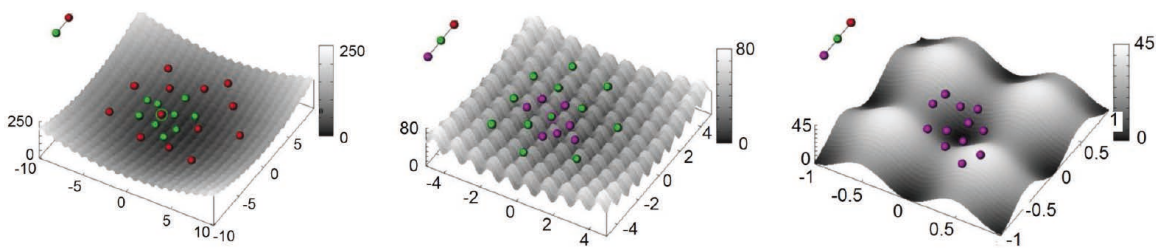

Figure 9. Left panel: root population (red) sprouting second level population (green). Middle panel: second level population (green) sprouting leaf population (pink). Right panel: leaf population (pink) performing local search.

To encode points from domain $\mathcal{D}$, we use a binary affine encoding [10]. Codes represent a set of identified parameters. Each code is assigned a fitness value - a misfit for a coded point. Fitness function is defined at each level separately (its accuracy depends on the level in the tree).

Different search accuracies are obtained by various encoding precisions and by changing the length of binary genotypes in demes of different orders. The root utilizes the shortest genotypes, while leaves utilize the longest ones. To obtain searchcoherency for demes at different levels, a hierarchical-nested encoding is used. First, the densest mesh of phenotypes in $\mathcal{D}$ is defined for demes at the $m$-th level. Afterwards, the meshes for lower order demes are recursively defined by selecting nodes from the previous ones. The maximum diameter of the mesh $\delta_{j}$ associated with demes of the order $j$ determines the search accuracy at $j$-th level of the HGS tree (see Figure 8). Defined mesh parameters satisfy $\delta_{m}<\ldots<\delta_{1}$.

In order to solve the particular class of inverse parametric problems, HGS may be coupled with the $h p$ adaptive goal-oriented FEM algorithm (see [4]). This strategy, called $h p$-HGS, consists of the estimation of the fitness value of each individual with the required accuracy depending on the level of the HGS tree. Direct problems at the root level are solved with the lowest accuracy and at leaf level - with the highest accuracy. Each problem solved by the $h p$-FEM algorithm consists of finding a logging curve for a particular set of parameters of formation layers with a given accuracy.

\section{Experiments}

\subsection{D DC resistivity logging measurements simulations}

We performed a simulation of the 3D DC borehole resistivity measurement problem using $h p$-HGS method with 3 levels. Parameters of the simulation are presented in Table 1. Sizes of populations were selected to balance the time of evaluating a single solution with search capabilities of a population. Code length for a single parameter was 15 on the first level, 21 on the second level, and 27 in the leaves.

The fitness value of each candidate solution $\omega$ (resistivity vector) was evaluated as the Euclidean norm of the difference between discrete representations of the reference 
Table 1

Parameters of the simulation in the DC case.

\begin{tabular}{lccc}
\hline & level 1 & level 2 & level 3 \\
\hline Population size & 12 & 6 & 4 \\
Code length & 45 & 63 & 81 \\
Mutation rate & 0.1 & 0.01 & 0.001 \\
Crossing rate & 0.5 & 0.5 & 0.5 \\
Accuracy & 0.1 & 0.01 & 0.001 \\
\hline
\end{tabular}

logging curve calculated with high accuracy and the logging curve computed by the self-adaptive goal-oriented $h p$-FEM algorithm for $\omega$ with accuracy depending on the level in HGS tree.

The accuracy (see last row in Table 1) corresponds to the maximum relative error decrement in the single $h p$-FEM step (see e.g. [20]) applied for solving direct problems at the particular HGS level.

Experiments were performed using the Linux cluster environment. The computation was stopped when the best leaf individual fitness was less than or equal to 0.10, which took 6 metaepochs.

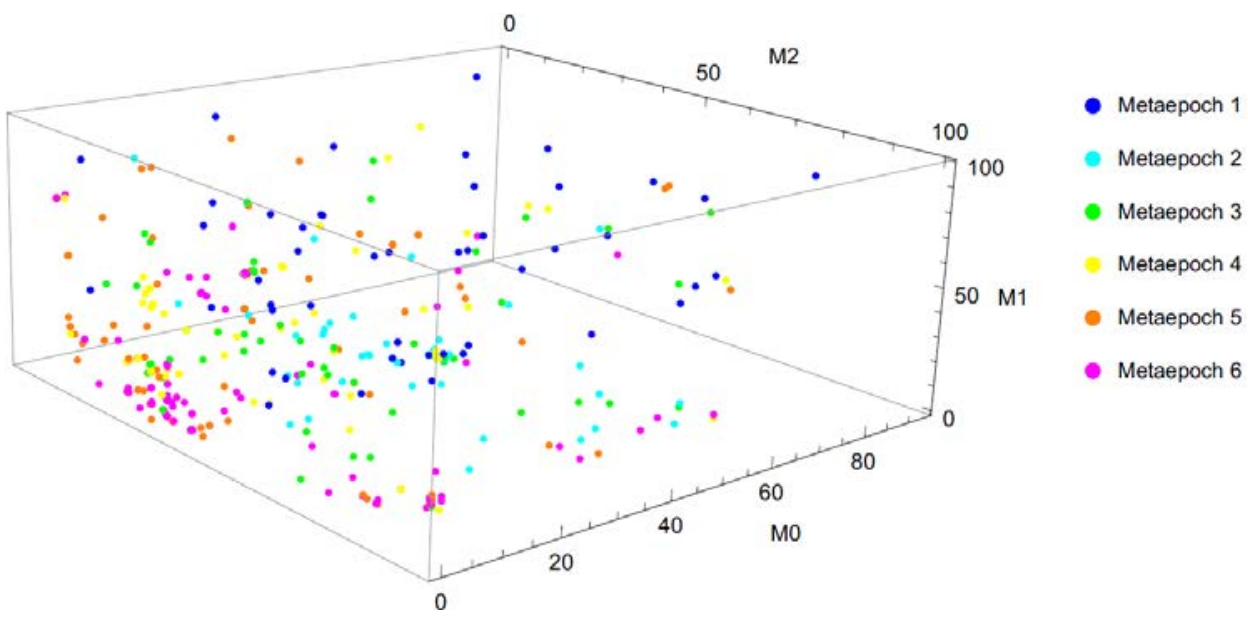

Figure 10. Convergence of $\omega_{0}, \omega_{1}$ and $\omega_{2}$ in DC case. Different colors represent individuals created in consecutive metaepochs.

Simulation results are presented in Figure 10. Figure 10 illustrates how the consecutive metaepochs concentrate closer and closer to the solution of the problem. Actually, the metaepochs converge to the left bottom border of the search domain, where $\omega_{0} \approx 1, \omega_{1} \approx 5$ and $\omega_{2}$ is within a range of $\approx(20,50)$. 


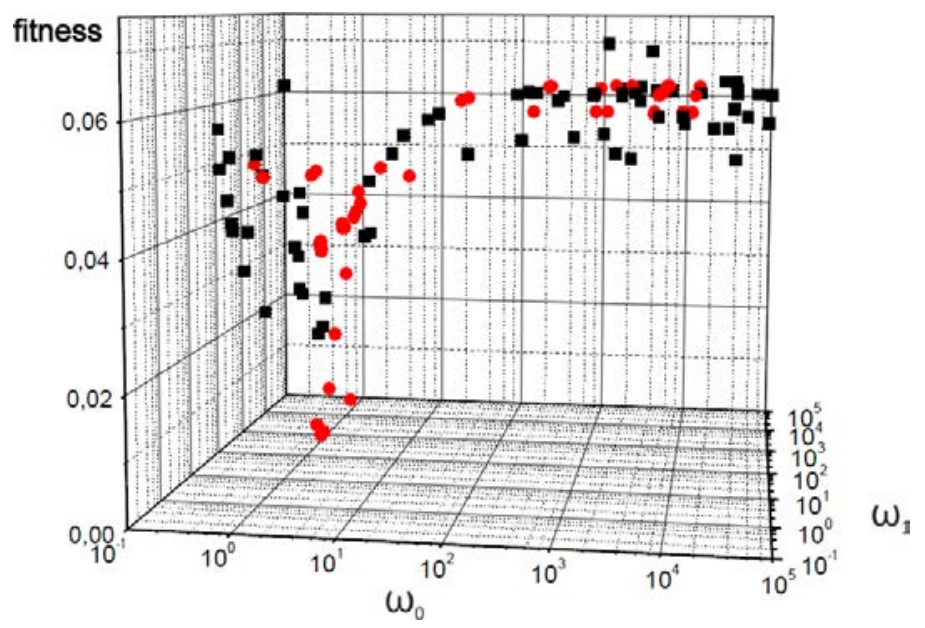

Figure 11. Found solutions for $\omega_{0}$ and $\omega_{1}$ in $\mathrm{AC}$ case. Black points represent root individuals and red represent leaf individuals.

The exemplary solution from the last metaepoch with the lowest fitness value of 0.10 was $\omega_{0}=0.995, \omega_{1}=3.605$ and $\omega_{2}=44.166$. Another solution with fitness value 0.2 was $\omega_{0}=0.789, \omega_{1}=3.578$ and $\omega_{2}=19.166$.

Results show that the fitness function is irregular in the neighborhood of the global minimum, and that the problem is more sensitive to $\omega_{0}$ than to other parameters. In other words, when we change the resistivity of the central layer within the range, the resulting logging curves will have similar shapes.

\subsection{D AC resistivity logging measurements}

We use data obtained from the logging tool working with the frequency $f=20 \mathrm{kHz}$.

Due to the very high computational cost of a single fitness evaluation in the 3D $\mathrm{AC}$ borehole resistivity measurement problem, we reduced the number of levels in HGS tree to 2 and modified the parameters of the simulation (see Table 2). Code length for a single parameter was 15 on the first level and 20 in leaves. Fitness values of candidate solutions were calculated analogously to the DC case. Simulation results are presented in Figure 11. The computation was stopped after 6 metaepochs.

Figure 11 differs from Figure 10 in the sense that colors do not correspond to metaepochs. All of the points represent individuals from the final tree of populations. The red points represents individuals found in the leaves of the tree. As we can see in Figure 11, some leaf populations contain individuals with low fitness values. Again, the algorithm was able to localize the $\omega_{0}$ parameter quite well; however, the $\omega_{1}$ parameter varies within the best fitted individuals, which implies that changing the conductivity of $\omega_{1}$ results in similar shape logging curves. Some examples of best fitted individuals are $\omega_{0}=1.12, \omega_{1}=74.89$, fitness 0.006331 or $\omega_{0}=1.03, \omega_{1}=10.40$, fitness 0.006975 . 
Table 2

Parameters of the simulation in the AC case.

\begin{tabular}{lcc}
\hline & level 1 & level 2 \\
\hline Population size & 6 & 4 \\
Code length & 30 & 40 \\
Mutation rate & 0.1 & 0.02 \\
Crossing rate & 0.5 & 0.5 \\
Accuracy & 10 & 1 \\
\hline
\end{tabular}

Both DC and AC results show the potential power of hp-HGS in resistivity identification and, similar to the DC case, the irregularity of fitness in the neighborhood of the global minimum.

\section{Conclusions}

In this paper, we presented $h p$-HGS hierarchical genetic search strategy combined with $h p$-adaptive finite element method solver. The strategy was utilized to solve the inverse problem related to the identification of formation layers based on borehole resistivity logging measurements. The $h p$-HGS strategy was tested on two cases; 1) the direct current case with zero frequency resistivity logging measurements tool and with three unknown formation layers, and 2) the alternate current case with $f=20 \mathrm{kHz}$ resistivity logging tool and with two unknown formation layers. The calculation converged to the expected solutions in both cases. The 30 percent disagreement between the inverse problem solution and the simulated measurements implies from the fact that the measurements are not sensitive to small variations of resistivities of certain formation layers. In other words, small changes in the resistivities of these layers imply the same shape of the logging curve. In future work, we plan to utilize hybrid stochastic / gradient strategies as well as perform measurements of the same formation layers with two different logging tools to eliminate inaccuracies in the numerical solutions.

\section{Acknowledgements}

The work presented in this paper has been supported by National Science Center grants no. NN519447 739, DEC-2011/03/B/ST6/01393 and DEC-2012/05/N/ST6/03433.

\section{References}

[1] Abubakar A., van den Berg P. M.: Nonlinear inversion in electrode logging in a highly deviated formation with invasion ising an oblique coordinate system. IEEE Transactions on Geoscience and Remote Sensing, vol. 38, pp. 25-38, 2000. 
[2] Abubakar A., Habashy T., Druskin V., Knizhnerman L., Davydycheva S.: A 3D parametric inversion algorithm for triaxial induction data. Geophysics, vol. 71, pp. G1-G9, 2006.

[3] Avdeev D. B., Kuvshinov A.V., Pankratov O. V., Newman G. A.: Threedimensional induction logging problems, Part 1: An integral equation solution and model comparisons. Geophysics, vol. 67, pp. 413-426, 2002.

[4] Barabasz B., Migórski S., Schaefer R., Paszyński M.: Multi-deme, twin adaptive strategy hp-HGS. Inverse Problems in Science and Engineering, vol. 19, pp. 3-16, 2011.

[5] Davydycheva S., Druskin V., and Habashy T.: An efficient finite-difference scheme for electromagnetic logging in 3D anisotropic inhomogeneous media. Geophysics, vol. 68(5), pp. 1525-1536, 2003.

[6] Denkowski Z., Migórski S., Papageorgiou N.: An Introduction to Nonlinear Analysis: Applications. Kluwer Academic/Plenum Publishers, 2003.

[7] Denkowski Z., Migórski S., Papageorgiou N.: An Introduction to Nonlinear Analysis: Theory. Kluwer Academic/Plenum Publishers, 2003.

[8] Druskin V.L., Knizhnerman L. A., Lee P.: New spectral Lanczos decomposition method for induction modeling in arbitrary 3-D geometry. Geophysics, vol. 64(3), pp. 701-706, 1999.

[9] Gajda-Zagórska E., Schaefer R., Smolka M., Paszyński M., Morytko R., and Pardo D.: A hybrid method for inversion of 3D DC resistivity logging measurements. submitted to Natural Computing, 2013.

[10] Holland J. H.: Adaptation in natural and artificial systems: an introductory analysis with applications to biology, control and artificial intelligence. MIT Press, 1992.

[11] Lu X., Alumbaugh D.L.: One-dimensional inversion of three-component induction logging in anisotropic media. SEG Expanded Abstract, vol. 20, pp. 376-380, 2001.

[12] Newman G. A., Alumbaugh D. L.: Three-dimensional induction logging problems, part 2: A finite-difference solution. Geophysics, vol. 67(2), pp. 484-491, 2002.

[13] Pardo D., Calo V.M., Torres-Verdín C., Nam M. J.: Fourier series expansion in a non-orthogonal system of coordinates for simulation of 3D DC borehole resistivity measurements. Computer Methods in Applied Mechanics and Engineering, vol. 197(1-3), pp. 1906-1925, 2008.

[14] Pardo D., Demkowicz L., Torres-Verdín C., and Paszynski M.:Simulation of resistivity logging-while-drilling (LWD) measurements using a self-adaptive goaloriented $h p$-finite element method. SIAM Journal on Applied Mathematics, vol. 66, pp. 2085-2106, 2006.

[15] Pardo D., Demkowicz L., Torres-Verdín C., Paszynski M.: A goal oriented $h p$ adaptive finite element strategy with electromagnetic applications. Part II: electrodynamics. Computer Methods in Applied Mechanics and Engineering, vol. 196, pp. 3585-3597, 2007. 
[16] Pardo D., Torres-Verdín C., Demkowicz L.: Simulation of multi-frequency borehole resistivity measurements through metal casing using a goal-oriented $h p$-finite element method. IEEE Transactions on Geosciences and Remote Sensing, vol. 44, pp. 2125-2135, 2006.

[17] Pardo D., Torres-Verdín C., Demkowicz L.: Feasibility study for two-dimensional frequency dependent electromagnetic sensing through casing. Geophysics, vol. 72, pp. F111-F118, 2007.

[18] Pardo D., Torres-Verdín C., Nam M. J., Paszynski M., Calo V. M.: Fourier series expansion in a non-orthogonal system of coordinates for simulation of $3 \mathrm{D}$ alternating current borehole resistivity measurements. Computer Methods in Applied Mechanics and Engineering, vol. 197, pp. 3836-3849, 2008.

[19] Pardo D., Torres-Verdín C., Paszynski M.: Simulation of 3D DC borehole resistivity measurements with a goal-oriented $h p$ finite element method. Part II: Through casing resistivity instruments. Computational Geosciences, vol. 12(1), pp. 83-89, 2008.

[20] Paszyński M., Pardo D., Torres-Verdżn C., Demkowicz L., V. C.: A parallel direct solver for the self-adaptive hp Finite Element Method. Journal of Parallel and Distributed Computing, vol. 70, pp. 270-281, 2010.

[21] Schaefer R., Kołodziej J.: Genetic search reinforced by the population hierarchy. In: Foundations of Genetic Algorithms 7, pp. 383-399. Morgan Kaufman Publisher, 2003.

[22] Vose M. D.: The Simple Genetic Algorithm. MIT Press, 1999.

[23] Wang T., Fang S.: 3-D electromagnetic anisotropy modeling using finite differences. Geophysics, vol. 66(5), pp. 1386-1398, 2001.

[24] Wang T., Signorelli J.: Finite-difference modeling of electromagnetic tool response for logging while drilling. Geophysics, vol. 69(1), pp. 152-160, 2004.

[25] Zhang J., Mackie R.L., Madden T. R.: 3-D resistivity forward modeling and inversion using conjugate gradients. Geophysics, vol. 60, pp. 1312-1325, 1995.

\title{
Affiliations
}

\author{
Ewa Gajda-Zagórska \\ AGH University of Science and Technology, Krakow, Poland, gajda@agh.edu.pl \\ Maciej Paszyński \\ AGH University of Science and Technology, Krakow, Poland, paszynsk@agh.edu.pl \\ Robert Schaefer \\ AGH University of Science and Technology, Krakow, Poland, schaefer@agh.edu.pl \\ David Pardo \\ University of The Basque Country, Bilbao, Spain, dzubiaur@gmail.com
}

Received: 6.04 .2013

Revised: 27.06 .2013

Accepted: 3.07.2013 